\title{
Why Do Mania and Suicide Occur Most Often in the Spring?
}

\author{
Chul-Hyun Cho and Heon-Jeong Lee ${ }^{\bowtie}$ \\ Department of Psychiatry and Chronobiology Institute, Korea University College of Medicine, Seoul, Republic of Korea
}

Symptoms of mental illness often become worse during the spring season. For patients with mood disorders, the phrase "spring fever" has a different meaning. Previous studies have reported that the incidence of mania peaks during the spring in both the Northern and Southern Hemispheres. ${ }^{1}$ Many studies have indicated that suicide rates also peak during the spring season. ${ }^{2}$ It has been suggested that the sudden increase in the duration and amount of sunlight is a possible cause of these phenomena. However, the mechanisms underlying the peak in incidence of mania and suicide during the spring season are unclear and remain to be elucidated.

All organisms on Earth must adapt to the 24-hour cycle of the Earth's rotation and the yearly seasonal cycle of the Earth's revolution. The daily light and dark cycle and the seasonal changes are a fundamental challenge to the necessary conditions for life; hence, all creatures have evolved a mechanism to adjust their physiology to these changes. The adjustment mechanism is the so-called circadian system. Circadian rhythms are physiological, mental, and behavioral changes that follow a roughly 24-hour cycle that regulates the approximate 24hour cycle of biological processes, such as sleep or hormone production. The rhythms tent to be not exactly 24 hours, but slightly longer than 24-hours. Daily environmental cues, such as light, resets people's circadian period back to the 24-hour day-night cycle. In mammals, circadian rhythms are generated by the suprachiasmatic nuclei (SCN) in a structure of the brain known as the hypothalamus with the day-night cycle as the primary environmental time cue that synchronizes the circadian system to the 24-hour day. Because the endogenous

Received: December 10, 2017 Accepted: December 20, 2017

$\triangle$ Correspondence: Heon-Jeong Lee, MD, PhD

Department of Psychiatry, Korea University College of Medicine, 73 Inchonro, Seongbuk-gu, Seoul 02841, Republic of Korea

Tel: +82-2-920-6721, Fax: +82-2-929-7679, E-mail: leehjeong@korea.ac.kr

(a) This is an Open Access article distributed under the terms of the Creative Commons Attribution Non-Commercial License (http://creativecommons.org/licenses/bync/4.0) which permits unrestricted non-commercial use, distribution, and reproduction in any medium, provided the original work is properly cited. circadian system can also be sustained without external time cues, misalignment between the endogenous circadian system and an individual's 24-hour environmental/behavioral cycles is possible, and it has been suggested that such misalignment can be related to health problems, especially mental illnesses. ${ }^{3}$ The circadian system regulates monoaminergic activity in the brain that controls mood through the local expression of circadian genes. Recently, several studies have reported that the occurrence of mood disorders is related to shifts and misalignment in the circadian rhythm. Moon et al. ${ }^{4}$ suggested that acute mood episodes are related to misalignment between the individual's endogenous circadian rhythms and the individual's physical environment. Acute manic episodes were associated with an average advance in the endogenous circadian rhythm over chronological time by 7 hours, although these could perhaps have resulted from an average of 17 hour clockwise delays. Individuals with mixed manias showed an average delay in their circadian cycle by more than 6 hours, whereas those with bipolar depression showed a 4 to 5 hour delay in their circadian cycle. It has been suggested that in patients with bipolar disorder, the circadian phase can be easily shifted by the disturbance of sleep-wake cycles and by unreliable zeitgebers (e.g., inappropriate artificial light). Cho et al. ${ }^{5}$ observed that significant delays in the circadian pattern of salivary cortisol levels, when an individual is exposed to bright light before bedtime, is related to subthreshold bipolarity. These findings suggest that severe circadian shifting and misalignment may represent a pathophysiological mechanism underlying acute mood episodes and suicide attempts in susceptible individuals.

\section{PHASE RESPONSE CURVE FOR LIGHT}

The phase response curve (PRC) for light is a curve describing the relationship between light exposure and phase shift in the circadian rhythm. ${ }^{6}$ The PRC determines the period of time at which exposure to light should be done in order to ad- 
vance (or delay) the circadian phase. Light exposure in the morning advances the rhythm, whereas light exposure at night delays it. The extent to which the rhythm is advanced or delayed depends on the time of the light exposure. The delay zone is the period spanning from two hours before the individual's usual bedtime to five hours after bedtime; exposure to light during this delay zone will delay the circadian phase. The PRC peaks at about five hours after the individual's usual bedtime, coinciding with the body temperature nadir; at this point in time, exposure to light will advance the circadian phase. Immediately after this peak, light exposure has its greatest phase-advancing effect. Factors influencing the strength of this effect include the intensity of the light, the length of the exposure, and the color of the light.

\section{ACUTE CIRCADIAN RHYTHM SHIFTING AND MISALIGNMENT: A POSSIBLE MECHANISM}

Why do mania and suicide occur most often in the spring? A possible mechanism underpinning these relationships is a misalignment between the endogenous and environmental circadian rhythms due to acute circadian rhythm shifting caused by the increased light exposure in the spring. The effects of light exposure are indicated by the PRC for light, as shown in the Figure 1. During the winter, the human circadian rhythm tends to be delayed because the sun rises late. Patients with winter depression have much delayed circadian rhythms, compared to the healthy population (see "Winter" in the Figure 1). During the onset of spring, the circadian rhythm of a healthy person advances in line with the earlier time of sunrise. On the other hand, in depressed patients with a delayed endogenous circadian rhythm, exposure to bright light early in the morning during the spring delays their circadian rhythm further, since the bright light occurs during the "delay zone" of the PRC for light. The significantly more delayed circadian rhythm is then able to induce a mixed/manic episode or emotional instability, leading to a possible suicide attempt in susceptible individuals.

A previous study reported that mixed states were induced by morning bright light therapy in women with bipolar depression, whereas midday light therapy had no effect; this suggests that abrupt phase shifts caused by bright light may be related to mixed states. ${ }^{7}$ It is possible that morning light therapy for patients with bipolar depression may induce further circadian delays because morning times may lie in the delay zone in the PRC for light in bipolar depression. ${ }^{6}$

This hypothesis provides excellent predictions regarding how best to prevent the increase of springtime mania and suicidal behavior. For example, it is important that susceptible individuals should be careful to prevent excessive circadian rhythm delays during the winter. Possible ways of achieving

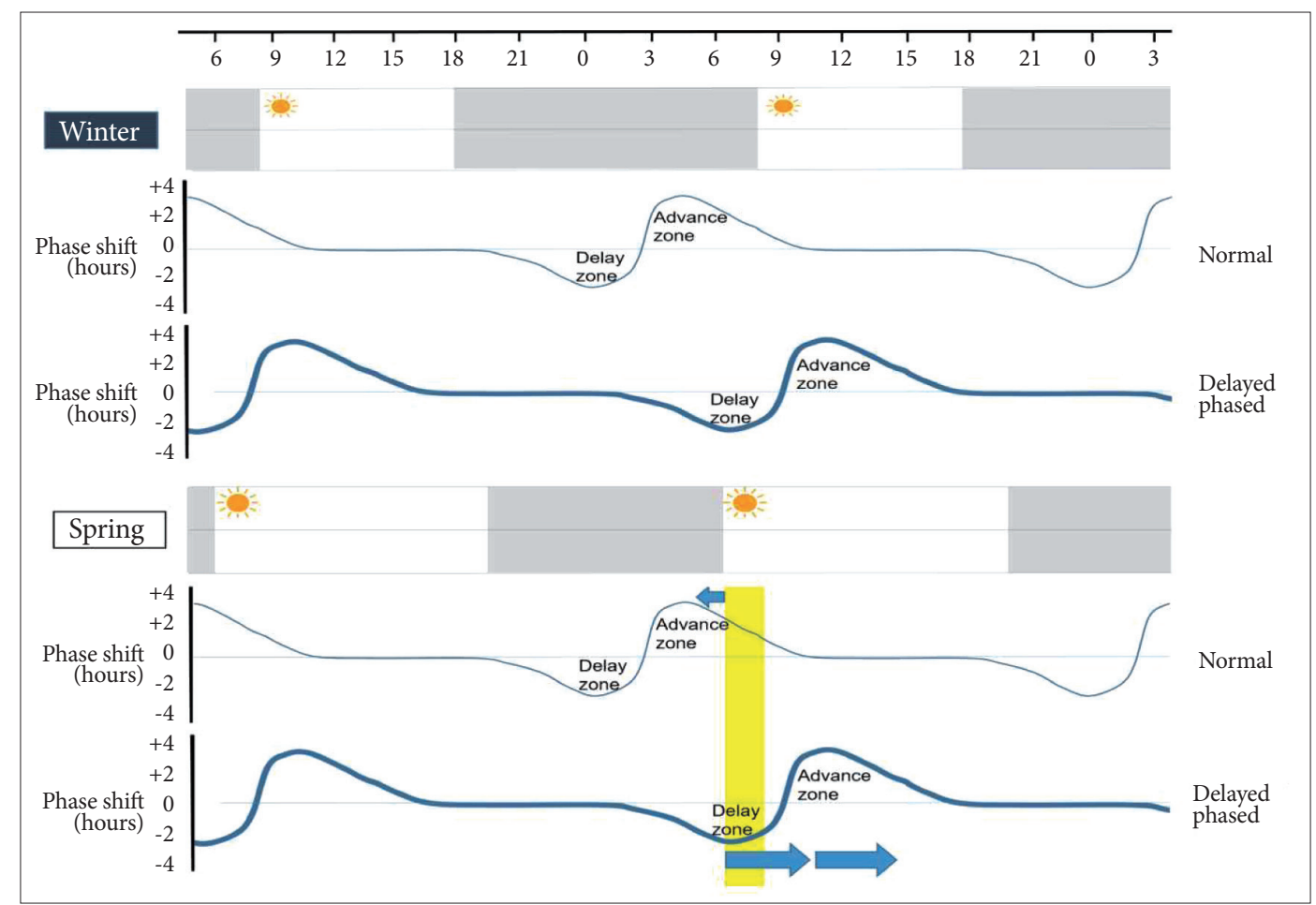

Figure 1. Extreme springtime delay in the circadian rhythm of individuals experiencing depression during the past winter compared with normal circadian rhythm advance. 
this include increasing outdoor activity in the morning and taking part in morning light therapy during the winter.

\section{Acknowledgements}

This study was supported from the Korea Health 21 R\&D Project funded by the Ministry of Health \& Welfare, Republic of Korea (HI14C3212 and HM14C2606).

\section{REFERENCES}

1. Lee HJ, Kim L, Joe SH, Suh KY. Effects of season and climate on the first manic episode of bipolar affective disorder in Korea. Psychiatry Res 2002;113:151-159.

2. Jee HJ, Cho CH, Lee YJ, Choi N, An H, Lee HJ. Solar radiation increases suicide rate after adjusting for other climate factors in South Korea.
Acta Psychiatr Scand 2017;135: 219-227.

3. Baron KG, Reid KJ. Circadian misalignment and health. Int Rev Psychiatry 2014;26:139-154.

4. Moon JH, Cho CH, Son GH, Geum D, Chung S, Kim H, et al. Advanced circadian phase in mania and delayed circadian phase in mixed mania and depression returned to normal after treatment of bipolar disorder. EBioMedicine 2016;11:285-295.

5. Cho CH, Moon JH, Yoon HK, Kang SG, Geum D, Son GH, et al. Molecular circadian rhythm shift due to bright light exposure before bedtime is related to subthreshold bipolarity. Sci Rep 2016;6:31846.

6. Khalsa SB, Jewett ME, Cajochen C, Czeisler CA. A phase response curve to single bright light pulses in human subjects. J Physiol Lond 2003; 549:945-952.

7. Sit D, Wisner KL, Hanusa BH, Stull S, Terman M. Light therapy for bipolar disorder: a case series in women. Bipolar Disord 2007:9:918-927. 